\title{
A Semi-Classical Trace Formula for Schrödinger Operators
}

\author{
R. Brummelhuis ${ }^{1}$ and A. Uribe ${ }^{2, \star}$ \\ 1 Mathematics Department, University of Wisconsin, Madison, WI 53706, USA \\ 2 Mathematics Department, University of Michigan, Ann Arbor, MI 48109 and \\ Institute for Advanced Study, Princeton, NJ 08540, USA
}

Received January 10, 1989; in revised form September 18, 1990

Abstract. Let $S_{h}=-\hbar \Delta+V$ on $\mathbb{R}^{n}$, with $V$ smooth. If $0<E^{2}<\liminf V(x)$, the spectrum of $S_{\hbar}$ near $E^{2}$ consists (for $\hbar$ small) of finitely-many eigenvalues, $\lambda_{j}(\hbar)$. We study the asymptotic distribution of these eigenvalues about $E^{2}$ as $\hbar \rightarrow 0$; we obtain semi-classical asymptotics for

$$
\sum_{j} f\left(\frac{\sqrt{\lambda_{j}(\hbar)}-E}{\hbar}\right)
$$

with $\hat{f} \in C_{0}^{\infty}$, in terms of the periodic classical trajectories on the energy surface $B_{E}=\left\{|\xi|^{2}+V(x)=E^{2}\right\}$. This in turn gives Weyl-type estimates for the counting function $\#\left\{j ;\left|\sqrt{\lambda_{j}(\hbar)}-E\right| \leqq c \hbar\right\}$. We make a detailed analysis of the case when the flow on $B_{E}$ is periodic.

\section{Table of Contents}

1. Introduction . . . . . . . . . . . . . 567

2. Statement of Results . . . . . . . . . . . . 569

3. On the Number of Eigenvalues Around a Given Energy Level. . . . . . . . 571

4. The Case of Periodic Flow . . . . . . . . . . . . . . . . . 575

5. The Trace Formula for Schrödinger Operators on $\mathbb{R}^{n}$. . . . 581

6. References . . . . . . . . . . . . . . . . . 584

\section{Introduction}

Consider the Schrödinger operator $S(\hbar)=-\hbar^{2} \Delta+V$ on $\mathbf{R}^{n}$, where $V \in C^{\infty}\left(\mathbf{R}^{n}\right)$, $V>0$. If $\underline{V}_{\infty}=\liminf _{|x| \rightarrow \infty} V(x)$, the intersection

$$
\operatorname{Spec} S(\hbar) \cap\left(-\infty, \underline{V}_{\infty}\right)
$$

* Research supported by NSF grants DMS-8610730 and DMS-8996279 
consists of eigenvalues $\lambda_{1}(\hbar) \leqq \lambda_{2}(\hbar) \leqq \ldots$, where we count each eigenvalue according to its (finite) multiplicity. In this paper we will analyze the distribution of the $\lambda_{j}(h)$ around a fixed energy level $E$ as $\hbar \rightarrow 0$, generalizing and complementing the results of Guillemin and Uribe [11] on compact manifolds. In particular, we will relate this asymptotic distribution to the classical dynamics of a particle with energy $E$ in a potential $V$.

Let us briefly describe the setting of [11], as it applies to $S(h)$ on a compact manifold. Further developing an idea to be found in Colin and Verdière [3], Guillemin and Uribe interpret $\hbar^{-1}$ as an eigenvalue of $i^{-1} \partial_{\theta}$ on the unit circle $S^{1}$, and make an analysis, in the spirit of [5], of the joint eigenvalue distribution $\left(\mu_{j, m}, m\right)$ of the two commuting operators $P=\left(-\Delta-V \partial_{\theta}^{2}\right)^{1 / 2}$ and $A=i^{-1} \partial_{\theta}$. Let $E>0$ be fixed and let $\varphi \in \mathscr{P}(\mathbb{R})$. Define

and

$$
\Upsilon_{1 / m}(\varphi)=\sum_{j} \varphi\left(\mu_{j}(m)-E m\right)
$$

$$
\Upsilon(\varphi)=\sum_{m} Y_{1 / m} e^{i m \theta}
$$

$\Upsilon(\varphi)$ can be interpreted as a trace, namely as $\operatorname{Tr}\left(\varphi(P-E A) e^{i \theta A}\right)$. The singularities of $\Upsilon(\varphi)$ are analyzed in [11], leading to asymptotics of $Y_{1 / m}(\varphi)$ as $\hbar^{-1}=m \rightarrow \infty$. These enable one to "see" spectrally the existence of periodic classical trajectories with a given energy, and to relate them quantitatively to the spectrum. This can of course be readily translated into a statement involving the eigenvalues $\lambda_{j}(h)$ $=m^{-2} \mu_{f}(m)^{2}\left(m=\hbar^{-1}\right)$ of $S(\hbar)$; the end result is a semi-classical version of the Selberg trace formula, in the spirit of the non-rigorous formula of Gutzwiller [6].

The principal aim of this paper is to extend these results to Schrödinger operators on $\mathbb{R}^{n}$. We will do this without introducing extraneous assumptions on the behavior of $V$ at infinity (which we would be forced to introduce if we tried to adapt the proofs of [11] to the case of $\mathbb{R}^{n}$ ). This is possible for Schrödinger operators because of the work of Helffer and Sjöstrand on exponential decay and localization of eigenvalue problems to wells, which we will apply. On $\mathbb{R}^{n}$, (1) gets replaced by (5) below; our main result is an asymptotic expansion of (5) as $\hbar \rightarrow 0$.

Another aim is to analyze the consequences of the asymptotics of (5) for the refined counting function

$$
N_{E, c}(\hbar)=\#\left\{j:\left|\sqrt{\lambda_{j}(\hbar)}-E\right| \leqq c \cdot \hbar\right\} .
$$

This will first be done in the setting of [11] (on compact manifolds) in Sect. 3, under the assumption that the flow is non-periodic a.e. on the energy surface $\{H=E\}$. This result will translate to estimates on (3) for the Schrödinger operator on $\mathbb{R}^{n}$. (There is some connection with [18], which deals with the error term in the Weyl estimate for the usual counting function; cf. the remarks after Theorem 2.2.)

In the opposite case, when the flow is periodic on $\{H=E\}$, we will derive in Sect. 4 (still on compact manifolds) a semi-classical analogue of the well-known results of $[4,5,22]$ for large eigenvalue asymptotics of a positive elliptic operator on a compact manifold all of whose bicharacteristics are closed. However, in the present set-up we will have to deal with $\exp (i t Q)$, with $Q$ being of real principal type rather than elliptic, under the assumption that all its null bicharacteristics are periodic.

Finally, in Sect. 5 we show how to pass from a compact manifold to $\mathbb{R}^{n}$. 
In the present semi-classical context, what has been mostly studied is the counting function $N_{E}^{\prime}(h):=\#\left\{j: \lambda_{j}(h) \leqq E\right\}$. For operators on $\mathbb{R}^{n}$ we refer to Chazarain [2], and the subsequent developments by Helffer-Robert [14, 15] and Petkov-Robert [18] (cf. also the books [19] by Robert and [12] by Helffer). These authors proceed along the lines of Hörmander [16], analyzing the singularity at $t=0$ of $\operatorname{Tr}[\exp (i t S(\hbar) / \hbar]$, treating $\hbar$ as a parameter. To be able to do this globally on $\mathbb{R}^{n}$ they need to introduce certain technical assumptions on the behavior of $\left|\partial^{\alpha} V(x)\right|$ for large $|x|$. It should be noted that their work applies to a more general class of operators (opérateurs $h$-admissibles). On the other hand, they do not analyze the singularities of the trace at $t$ 's different from 0 . Furthermore, our analysis is finer in that we work on a fixed energy shell $\{H=E\}$; our method uses standard Fourier integral operator theory.

Finally, let us note that in this paper, as in $[11], \hbar^{-1}$ will only range over the set $\left\{m^{-1}: m \in \mathbb{N}\right\}$. This is of course a restriction, but note that, at least in the case of a periodic classical trajectory, the Bohr-Sommerfeld quantization condition gives a good philosophical reason to restrict $\hbar$ to the inverse of an arithmetical sequence; cf. Corollary 2.5.

After the results of this paper were obtained, we learned from André Voros that he had considered an object very similar to (2) in [21]. In fact, our $Y(\varphi)$ is a smoothed-out version of his $\Theta\left(R^{2}, s\right)$, if we put $s=i \theta$. Voros bases his analysis on Gutzwiller's non-rigorous trace formula [6], and obtains a result formally analogous to our results on the singularities of $r(\varphi)$.

\section{Statement of Results}

We now formulate the main results of this paper. Fix $E>0$ such that $E^{2}<\underline{V}_{\infty}$, pick $0<\varepsilon<1$, and consider the following set of eigenvalues:

$$
J_{E, \varepsilon}(\hbar)=\left\{j:\left|\sqrt{\lambda_{j}(\hbar)}-E\right| \leqq h^{1-\varepsilon}\right\} .
$$

This is a finite set if $\hbar$ is sufficiently small. Define

$$
\Upsilon_{\hbar}(\varphi)=\sum_{j \in J_{E, \mathrm{c}(h)}} \varphi\left(\frac{\sqrt{\lambda_{j}(\hbar)}-E}{\hbar}\right),
$$

where $\varphi \in \mathscr{S}(\mathbb{R})$. Note that if $\underline{V}_{\infty}<\infty$, summation over all $j$ in (5) would not make sense in general. Also, in case of a compact manifold, the difference between (5) and the analogous sum over all $j$ is $O\left(\hbar^{\infty}\right)$; cf. Lemma 5.2 below.

Let

$$
H(x, \xi)=\sqrt{|\xi|^{2}+V(x)} .
$$

Considered as a Hamiltonian on $T^{*} \mathbb{R}^{n}=\mathbb{R}^{n} \oplus \mathbb{R}^{n}$, it generates a Hamilton flow which we denote by $\phi_{i}$. Let $E$ be as above, and let $B_{E}$ be the energy surface $\{H=E\}$. Notice that, since $E^{2}<\underline{V}_{\infty}, B_{E}$ is compact. We will furthermore assume that $E$ is a regular value of $H$, so $B_{E}$ is a manifold; we denote its Liouville measure by $\operatorname{Vol}\left(B_{E}\right)$. If $\gamma$ is a periodic trajectory of $\phi_{t}$, we let

$$
S_{\gamma}=\int_{\gamma} \xi d x
$$

denote its action. 
Finally, we will need to assume that $\phi_{t}$ restricted to $B_{E}$ is a clean flow. [We recall the definition for convenience of the reader: A smooth flow $\phi_{t}$ on a manifold $Y$ is said to be clean iff the set

$$
\mathscr{P}=\left\{(T, y) \in \mathbb{R} \times Y ; \phi_{T}(y)=y\right\}
$$

is a smooth submanifold of $\mathbb{R} \times Y$, and at each $(T, y) \in \mathscr{P}$ the tangent space to $\mathscr{P}$ is the set of all $(\tau, v) \in \mathbb{R} \times T_{y} Y$ such that

$$
\tau \Xi_{y}+d\left(\phi_{T}\right)_{y}(v)=v,
$$

where $\Xi$ is the infinitesimal generator of the flow. If the set of periods of the flow is discrete, cleanness of the flow is equivalent to the following: for every period $T$, the set $\mathscr{P}_{T}=\left\{y \in Y ; \phi_{T}(y)=y\right\}$ is a manifold, and at each $y \in \mathscr{P}_{T}$ its tangent space is identical with the set of fixed vectors of $d\left(\phi_{T}\right)_{y}$.] We can now state:

Theorem 2.1. Assume $E$ is a regular value of $H$ and the restriction of the flow $\phi_{t}$ to $B_{E}$ is clean. Then, for each function $\varphi$ such that $\hat{\varphi} \in C_{0}^{\infty}(\mathbb{R})$, there is an asymptotic expansion

$$
\Upsilon_{\bar{n}}(\varphi) \sim \sum_{j=0}^{\infty} c_{j}(h, \phi) \hbar^{j-d}
$$

as $\hbar=1 / m$ tends to zero. Each coefficient $c_{j}$ is $O(1)$ in $\hbar$. Moreover, we can say the following about the leading coefficient in the expansion, $c_{0}$, and the degree $d=d(\varphi)$ :

(i) If 0 is the only period in $\operatorname{supp}(\hat{\varphi})$, then $d=n-1$ and

$$
c_{0}(h, \varphi)=\frac{\operatorname{Vol}\left(B_{E}\right)}{(2 \pi)^{n}} \hat{\varphi}(0) .
$$

(ii) More generally, assume that there is a unique period.T. of the flow in the support of $\hat{\varphi}$. Let $Y_{1}, \ldots, Y_{r}$ be the connected components of $\left\{x \in B_{E} ; \phi_{T}(x)=x\right\}$ of maximal dimension, and let this dimension by $k$. Then $d=k-1$, and $\forall j$ there is a density $v_{j}$ on $Y_{j}$, defined in terms of the classical dynamics, such that

$$
c_{0}(\hbar, \varphi)=\hat{\varphi}(T) \sum_{j=1}^{r} e^{\pi i \sigma_{j} / 2} e^{i \hbar^{-1} S_{j}} \int_{Y_{j}} v_{j},
$$

where $\sigma_{j}$ is the (common) Maslov index of the trajectories in $Y_{j}$ and $S_{j}$ their (common) action.

Remark. The densities $v_{j}$ are described in [11, Sect. 4], where it is also described what the statement is if the flow is clean but the period spectrum is not discrete. This situation can actually occur; however, if the flow is clean, the set of numbers $\int p d q$ where $\gamma$ ranges over all the periodic trajectories in $B_{E}$ is discrete, which is what is fundamental for the proof of the theorem.

If there is a unique periodic trajectory $\gamma \subset B_{E}$ whose period $T_{\gamma}$ is in the support of $\hat{\varphi}$, then $d=0$ and the density in (ii) can be computed explicitly. This results in:

$$
c_{0}(\hbar, \varphi)=\frac{T_{\gamma}^{\#} e^{\pi i \sigma_{y} / 2}}{2 \pi\left|I-P_{\gamma}\right|^{1 / 2}} e^{i \hbar-1} S_{\gamma} \hat{\varphi}\left(T_{y}\right),
$$

where $T_{\gamma}^{\#}, P_{y}$, and $\sigma_{\gamma}$ are respectively the primitive period, the Poincare map and the Maslov index of $\gamma$. 
Theorem 2.2. Assume that $E<\sqrt{\underline{V}_{\infty}}$ is a regular value of $H$, and that the set of periodic points in $B_{E}$ has Liouville measure zero. Then

$$
\lim _{h=1 / m \rightarrow 0} \hbar^{n-1} N_{E, c}(\hbar)=2 c \cdot \operatorname{Vol}\left(B_{E}\right) /(2 \pi)^{n} .
$$

Formally, this result also follows from Petkov and Robert [18], who prove under the assumption of a.e. non-periodicity of the flow that the error term in the Weyl formula for the usual counting function $\#\left\{j: \lambda_{j}(\hbar) \leqq E\right\}$ is $o\left(\hbar^{-(n-1)}\right)$. However, to make this rigorous one would need to assume also non-periodicity a.e. of the flow on nearby energy surfaces, thereby obtaining a weaker result. Moreover, in our set-up, 2.2. is a straightforward consequence of the trace formula, and as such also holds in the circular symmetry setting of [11], which is more general than the semi-classical one.

At the other extreme, we have:

Theorem 2.3. Assume that $B_{E}$ is connected and that all points on it are periodic with a common minimal period $T>0$. Let $\alpha=\int_{\gamma} p d q$ denote the common action of the simple trajectories on $B_{E}$, and let $\sigma \in \mathbb{Z}$ be the common Maslov index of these trajectories. Suppose that $\alpha \in 2 \pi \mathbb{Z}$. Then there exist constants, $C_{1}, C_{2}>0$ such that for $\hbar$ sufficiently small and of the form $\hbar=m^{-1}, m \in \mathbb{Z}$ :

$$
\left\{j ;\left|\sqrt{\lambda_{j}(\hbar)}-E-\hbar \pi \sigma / 2 T\right| \leqq C_{1} \hbar\right\}=\left\{j ;\left|\sqrt{\lambda_{j}(\hbar)}-E-\hbar \pi \sigma / 2 T\right| \leqq C_{2} \hbar^{2}\right\} .
$$

Moreover, there exists a polynomial $p$ of degree $n-1$ and leading coefficient equal to $\operatorname{Vol}\left(B_{E}\right) /(2 \pi)^{n}$ such that for $\hbar=m^{-1}$ small enough the cardinality of (13) is equal to $\mathfrak{p}(m)$.

Corollary 2.4. With the same hypotheses as Theorem 2.3, for all $c<C_{1}$,

$$
\lim _{\hbar=1 / m \rightarrow \infty} h^{n-1} N_{E+\hbar \pi \sigma / 2 T, c}(\hbar)=\operatorname{Vol}\left(B_{E}\right) /(2 \pi)^{n} \text {. }
$$

This corollary should be compared with Theorem 2.2. By a rescaling of (the proof of) Theorem 2.3 one obtains:

Corollary 2.5. If the common action $\alpha$ is not in $2 \pi \mathbb{Z}$, Theorem 2.3 and its corollary remain true if we let $\hbar$ range over $\{\alpha / 2 \pi m ; m \in \mathbb{Z}\}$.

Note that the condition relating $\alpha$ and $\hbar$ in 2.5 is the Bohr-Sommerfeld quantization condition, $\alpha=2 \pi m \hbar$, without Maslov corrections. The latter are of course present in the counting function in Corollary 2.4 .

\section{On the Number of Eigenvalues around a Given Energy Level}

In this section we will prove Theorem 2.2 on a compact manifold. With an eye toward other applications we will do this in the more general setting of [11]. Thus let $M$ be a compact, $n$-dimensional manifold, and let $P$ and $A$ be two commuting, self-adjoint classical first-order $\Psi D O$ 's, such that $P$ is positive and elliptic and $\operatorname{Spec}(A)$ is contained in $\mathbb{Z}$, so that $\theta \mapsto e^{i \theta A}$ is a representation of the circle group. For each $m \in \mathbb{Z}$, let

$$
\mu_{1}(m) \leqq \mu_{2}(m) \leqq \cdots
$$


be the eigenvalues (each counted according to its multiplicity) of the restriction of $P$ to the $m$-eigenspace of $A$. Fix $E>0$. If $\varphi \in \mathscr{S}(\mathbb{R})$, define $\Upsilon_{1 / m}(\varphi)$ and $\Upsilon(\varphi)$ by (1) and (2).

We will assume that the action of $S^{1}$ on $T^{*}(M)$ induced by the Hamilton flow of $a$ is free, so that

$$
B=\left\{x \in T^{*}(\tilde{M})-0: a(x)=1\right\} / S^{1}
$$

is a manifold, which is in fact symplectic. Note that $p$ descends to a function $H$ on $B$, since $\{p, a\}=0$. Let $\phi_{t}$ denote the Hamilton flow of $H$ on $B$ and let

$$
B_{E}=\{b \in B: H(b)=E\} \text {. }
$$

Note that $\{a=1\} \rightarrow B$ is a principal circle bundle on which the canonical one form of $T^{*}\left(\mathbb{R}^{\eta}\right)$ induces a natural connection. If $\gamma$ is a closed curve in $B$, we let $\exp \left(i \int_{\gamma} \alpha\right)$ denote the holonomy of $\gamma$ with respect to this connection. The logarithm of the holonomy of $\gamma$ will be interpreted as the action of $\gamma$.

For the purposes of Sect. 1 of this paper, the relevant example of this situation is the following: Let $M$ be of the form $M=\tilde{M} \times S^{1}, \tilde{M}$ a compact Riemannian manifold with Laplace-Beltrami operator $\Delta$, and $A=i^{-1} \partial_{\theta}, P=\left(-\Delta-V \partial_{\theta}^{2}\right)^{1 / 2}$, with $V>0$ in $C^{\infty}(\tilde{M})$. In this case $B$ is just $T^{*}(\tilde{M})$ and $\int_{\gamma} \alpha$ is just the integral over $\gamma$ of the canonical one-form $\xi d x$ of $T^{*}(\tilde{M})$.

The main result of [11] implies that the singularities of $Y$ (considered as a periodic distribution on $\mathbb{R}$ ) are amongst the values of the actions of the closed trajectories of $\phi_{t}$ :

Theorem 3.1 (cf. [11, Theorem 4.3, Theorem 4.7]). (i) If a point $z \in S^{1}$ is in the singular support of $Y$, there is a periodic trajectory $\gamma$ of $\phi_{t}$ on $B_{E}$ whose period is in the support of $\hat{\varphi}$ (the Fourier transform of $\varphi$ ) and such that

$$
z=\exp \left(i \int_{\gamma} \alpha\right)
$$

(ii) If $E$ is a regular value of $H$ and the flow $\phi_{t}$ on $B_{E}$ is clean, then there is an asymptotic expansion

$$
\Upsilon_{1 / m}(\varphi) \sim \sum_{j \geqq 0} c_{j}(m, \varphi) m^{d(\varphi)-j}, \quad m \rightarrow \infty,
$$

with $c_{j}(m, \varphi)$ as in Theorem 2.1, if we put $\hbar=m^{-1}$.

In particular, $c_{0}(m, \varphi)=(2 \pi)^{-n}\left(\operatorname{Vol} B_{E}\right) \hat{\varphi}(0)$ if 0 is the only period in supp $\hat{\varphi}$. We will show that this implies a Weyl-type estimate. Let

$$
N_{E, c}(m)=\#\left\{j:\left|\lambda_{j}(m)-m E\right| \leqq c\right\} .
$$

Theorem 3.2. Assume that $E$ is a regular value of $H$, and that the set of periodic points on $B_{E}:=H^{-1}(E)$,

$$
\left\{b \in B_{E} \mid \exists T>0, \phi_{T}(b)=b\right\}
$$

has Liouville measure zero. Then

$$
\lim _{m \rightarrow \infty} m^{-(n-1)} N_{E, c}(m)=(2 \pi)^{n-1} \cdot 2 c \cdot \operatorname{Vol}\left(B_{E}\right) .
$$

The proof of this theorem is based on the observation that the hypothesis on the flow in 3.2 implies the following: 
Let $\varphi$ be a Schwartz function on the real line such that its Fourier transform $\hat{\varphi}$ has compact support. Then, under the assumptions of Theorem 3.2,

$$
\sum_{j \geqq 1} \varphi\left(\lambda_{j}(m)-m E\right)=(2 \pi)^{n-1} \hat{\varphi}(0) \operatorname{Vol}\left(B_{E}\right) m^{n-1}+o\left(m^{n-1}\right) .
$$

If we assume that $\phi_{t}$ is a clean flow this follows immediately from Theorem 3.1 since the contributions coming from the periods $T>0$ are of strictly lower order in $m$. Actually, (22) remains true without any cleanness assumption on the flow, as can be shown directly by a stationary phase argument.

To prove (21) from (22) we will use the following two lemmas.

Lemma 3.3. Let $0<E_{1}<E_{2}$ be such that $\left[E_{1}, E_{2}\right]$ consists entirely of regular values of $H$. Let $a>0$. Then there exists a constant, $C=C\left(a, E_{1}, E_{2}\right)$ such that for all $E \in\left[E_{1}, E_{2}\right]$,

$$
\#\left\{j|| \lambda_{j}(m)-m E \mid \leqq a\right\} \leqq C m^{n-1} .
$$

Proof. It follows trivially from (22) that the left-hand side of (23) is $O_{E}\left(m^{n-1}\right)$. The point of this lemma is that the estimate is locally uniform in $E$, which follows from inspection of the proof of (22).

Fix $c>0$, and let $\chi$ be the characteristic function of the interval $[-c, c]$. Let $\psi$ be a Schwartz function on the line such that $\psi \geqq 0, \hat{\psi}(0)=1$, and the support of $\hat{\psi}$ is compact. Define, for each $\delta>0$,

$$
\begin{gathered}
\psi_{\delta}(x)=\delta^{-1} \psi\left(\delta^{-1} x\right), \\
\varphi_{\delta}=\psi_{\delta} * \chi .
\end{gathered}
$$

The functions $\varphi_{\delta}$ converge to $\chi$ as $\delta \rightarrow 0$. We will apply (22) to each $\varphi_{\delta}$. The following lemma is the main technical step in the proof of theorem and is analogous to Lemma 3.3 of [5]. Since the proof is slightly more involved [because (23) does not hold globally uniformly in $E]$ we give the proof in full. As customary, the precise values of the constants $C, C_{N}$ below may differ from line to line.

Lemma 3.4. Suppose $0<\gamma<c$. Then, for all $N$ sufficiently large, there exist constants $C=C(N)$ and $K=K(N)$ such that

$$
\begin{aligned}
N_{E, c-\gamma}(m)\left(1-C(\delta / \gamma)^{N}\right) & \leqq \sum_{j \geqq 1} \varphi_{\delta}\left(\lambda_{j}(m)-m E\right) \\
& \leqq N_{E, c+\gamma}(m)+K(\delta / \gamma)^{N} m^{n-1} .
\end{aligned}
$$

Proof. Since

$$
\varphi_{\delta}(x)=\int_{(x-c) / \delta}^{(x+c) / \delta} \psi(y) d y
$$

it follows

$$
\begin{gathered}
\min _{|x| \leqq c-\gamma} \varphi_{\delta} \geqq \int_{-\gamma / \delta}^{\gamma / \delta} \psi(y) d y \\
=1-\int_{|y| \geqq \gamma / \delta} \psi(y) d y \geqq 1-C(\delta / \gamma)^{N},
\end{gathered}
$$

where the last inequality holds by the rapid decrease of $\psi$. This proves the first inequality in (26). 
To simplify the notation in the proof of (27), write $x_{j}=\lambda_{j}(m)-m E$ and $c^{\prime}=c+\gamma$. Then

$$
\sum_{j \geqq 1} \varphi_{\delta}\left(x_{j}\right)=\sum_{j:\left|x_{j}\right|<c^{\prime}} \varphi_{\delta}\left(x_{j}\right)+\sum_{j:\left|x_{j}\right| \geqq c^{\prime}} \varphi_{\delta}\left(x_{j}\right)
$$

The first sum is dominated by $N_{E, c^{\prime}}(m)$. To estimate the second sum, we proceed as follows. First, let $\varepsilon>0$ be such that the interval $[E-\varepsilon, E+\varepsilon]$ consists entirely of regular values of $H$ ( $\varepsilon$ exists since the set of regular values is open by compactness of $M$ ). Next write the second sum on the right-hand side of (31) as a sum $I+I I$, where

and

$$
I=\sum_{k=0}^{[e m]} \sum_{c^{\prime}+k \leqq\left|x_{j}\right|<c^{\prime}+k+1} \varphi_{\delta}\left(x_{j}\right)
$$

$$
I I=\sum_{k=[\varepsilon m]+1}^{\infty} \sum_{c^{\prime}+k \leqq\left|x_{j}\right|<c^{\prime}+k+1} \varphi_{\delta}\left(x_{j}\right) .
$$

To estimate $I$, first note that

$$
\begin{aligned}
& \#\left\{j ; c^{\prime}+k \leqq\left|x_{j}\right|<c^{\prime}+k+1\right\} \\
& \leqq \#\left\{j:\left|\lambda_{j}(m)-m(E+k / m)\right| \leqq c^{\prime}+1 \text { or }\left|\lambda_{j}(m)-m(E-k / m)\right| \leqq c^{\prime}+1\right\},
\end{aligned}
$$

which is $\leqq C m^{n-1}$ if $k \leqq[\mathrm{gm}]$, by Lemma 3.3 . Hence

$$
\begin{aligned}
|I| & \leqq C m^{n-1} \sum_{k=0}^{[\varepsilon m]} \max _{c^{\prime}+k \leqq|x| \leqq c^{\prime}+k+1}\left|\varphi_{\delta}(x)\right| \\
& \leqq C m^{n-1} \sum_{k=0}^{\infty} \int_{|y| \geqq(k+\gamma) / \delta} \psi(y) d y \\
& \leqq C_{N} m^{n-1} \sum_{k=0}^{\infty}\left(\frac{\delta}{k+\gamma}\right)^{N},
\end{aligned}
$$

where the last inequality uses the rapid decrease of $\psi$. If in the last series we factor out $(\delta / \gamma)^{N}$ and sum the rest, we obtain

$$
I \leqq C_{N} m^{n-1}\left(\frac{\delta}{\gamma}\right)^{N}
$$

(Since in the proof of Theorem 3.2 we will let $\gamma \rightarrow 0$, we may assume and use here that $\gamma<1$.) We now turn to the estimate of $I I$. By the rough estimate (78) of Sect. 5, we see that, for some $N_{0}$ and some constants $C$,

$$
\#\left\{j ;\left|\lambda_{j}(m)-m(E \pm k / m)\right| \leqq c^{\prime}\right\} \leqq C m^{N_{0}}(E+k / m)^{N_{0}} \leqq C m^{N_{0}} k^{N_{0}},
$$

and therefore, using once again the rapid decrease of $\psi$, if $N$ is large enough

$$
\begin{aligned}
|I I| & \leqq C_{N} m^{N_{0}} \sum_{k=[\varepsilon m]+1}^{\infty}\left(\frac{\delta}{k+\gamma}\right)^{N} k^{N_{0}} \\
& \leqq C_{N} m^{N_{0}}\left(\frac{\delta}{\gamma}\right)^{N} \sum_{k=[\varepsilon m]+1}^{\infty}\left(\frac{1}{k}\right)^{N-N_{0}} \\
& \leqq C_{N}\left(\frac{\delta}{\gamma}\right)\left(\frac{1}{\varepsilon}\right)^{N-N_{0}+1} m^{2 N_{0}-N+1}
\end{aligned}
$$


Choosing $N$ such that $2 N_{0}-N+1 \leqq n-1$ and adding the estimates for $I$ and $I I$ completes the proof of (26).

Proof of Theorem 3.2. Let $0<\gamma<$ c. Apply (22) and (26) to $\varphi_{\delta}$. Then, if $V$ is the Liouville measure of $B_{E}$, we have

$$
N_{E, c-\gamma}(m)\left(1-C_{N} \delta^{N}\right) \leqq(2 \pi)^{n-1} 2 c V m^{n-1}+o_{\delta}\left(m^{n-1}\right)
$$

and

$$
N_{E, c+\gamma}(m)+C_{N}^{\prime} \delta^{N} m^{n-1} \geqq(2 \pi)^{n-1} 2 c V m^{n-1}+o_{\delta}\left(m^{n-1}\right) .
$$

Dividing by $m^{n-1}$ and letting $m \rightarrow \infty$ first and $\delta \rightarrow 0$ afterwards, we obtain

$$
\limsup _{m \rightarrow \infty} m^{-(n-1)} N_{E, c-\gamma} \leqq(2 \pi)^{n-1} 2 c V \leqq \liminf _{m \rightarrow \infty} m^{-(n-1)} N_{E, c+\gamma}(m) .
$$

Now just apply (45) with $c$ replaced by $c+\gamma$ and then with $c$ replaced by $c-\gamma$, and finally let $\gamma \rightarrow 0$.

\section{The Case of Periodic Flow}

$A$. We first discuss a general theorem on operators of real principal type. Let $Q$ be any first-order, self-adjoint pseudodifferential operator of real principal type on a compact manifold $M$ such that all its null bicharacteristics are periodic of minimal period $T>0$. Let $q$ be the principal symbol of $Q$, and assume for simplicity that its subprincipal symbol is zero. Let $\sigma$ be the Maslov index of the null bicharacteristics, and $\Sigma=q^{-1}(0)$ the characteristic variety.

Theorem 4.1. There exist an orthogonal projection, $\Pi$, and a self-adjoint pseudodifferential operator of order $(-1), R$, such that $[\Pi, Q]=0=[\Pi, R]$, and if we set $\mathscr{Q}=\left(Q-\frac{\pi \sigma}{2 T} I-R\right)$, we have

$$
\mathscr{Q} \circ \Pi=0 \text {. }
$$

Moreover, there is a bounded operator, $L$, such that

$$
L \circ \mathscr{Q}=I-\Pi+S \text {, }
$$

where $S$ is a smoothing operator. The projector $\Pi$ is a Fourier integral operator associated with the characteristic relation

$$
\mathscr{C}=\{(x, y) \in \Sigma \times \Sigma ;(x, y) \text { are in the same bicharacteristic strip }\},
$$

while $L$ is in the class $I^{-1 / 2,-1 / 2}(M \times M ; \Delta, \mathscr{C})$ of Guillemin and Uhlmann, [10], where $A$ is the diagonal in $T^{*} M \times T^{*} M \backslash 0$. In the circular symmetry setting, all operators can be chosen to commute with the $S^{1}$ action.

The projector $\Pi$ will be a smoothed-out version of the operator

$$
\frac{1}{T} \int_{0}^{T} e^{i t Q} d t
$$

which is the exact answer in case $Q$ is $(-i)$ times the infinitesimal generator of a circle action on $M$.

The canonical relation (48) has the property that composed with itself yields itself, and the composition is clean in the sense of FIO theory [8]. Thus the Fourier 
integral operators associated to it form a ring $\mathscr{R}$; in fact $\mathscr{R}$ is a $*$-algebra over the ring of pseudodifferential operators. We seek to construct $I I$ symbolically in this algebra. We will need to discuss the non-commutative symbol calculus of operators in $\mathscr{R}$, introduced by Guillemin and Sternberg in [8], modified to include Maslov factors. Due to lack of space, we will be sketchy.

The circle group $S_{T}^{1}=\mathbb{R} / T \mathbb{Z}$ acts freely on $\Sigma$ by the Hamilton flow of $q$; let $W=\Sigma / S_{T}^{1}$ and $\pi: \Sigma \rightarrow W$ the natural projection. $W$ has a natural symplectic structure. $\forall w \in W$, let $F^{w}=\pi^{-1}(w)$; this is an affine circle. Let $\mathscr{L}^{w} \rightarrow F^{w}$ be the flat complex line bundle defined by the character of $\pi_{1}\left(F^{w}\right)=\mathbb{Z}$ which to the obvious generator associates $e^{-i \pi \sigma T / 2}$.

Lemma 4.2. $\forall(x, y) \in \mathscr{C}$ there is a natural identification

$$
\mathscr{S}_{(x, y)} \cong\left|T_{x} F^{w}\right|^{1 / 2} \otimes \mathscr{L}_{x}^{w} \otimes\left|T_{y} F^{w}\right|^{1 / 2} \otimes \mathscr{L}_{y}^{w},
$$

where $w=\pi(x)=\pi(y)$, and $\mathscr{S}=|T \Sigma|^{1 / 2} \otimes \mathscr{M}_{\mathscr{C}}$ is the symbol bundle of $\mathscr{C}$, tensor product of its half-density and Maslov bundles. This identification depends smoothly on $(x, y)$.

For the construction of this identification ignoring Maslov factors, see [8]. We hope to provide the details of the Maslov factors (in greater generality) elsewhere.

The space of smooth sections of the bundle $\left|T F^{w}\right|^{1 / 2} \otimes \mathscr{L}^{w}$ has a natural preHilbert space structure. By (50), a symbol s on $\mathscr{C}$ (i.e. a section of $\mathscr{S}$ ) defines, for each $w \in W$, a smoothing operator

$$
s_{w}: C^{-\infty}\left(\left|T F^{w}\right|^{1 / 2} \otimes \mathscr{L}^{w}\right) \rightarrow C^{\infty}\left(\left|T F^{w}\right|^{1 / 2} \otimes \mathscr{L}^{w}\right) .
$$

Proposition 4.3. The correspondence $s \mapsto\left\{s_{w} ; w \in W\right\}$ between symbols on $\mathscr{C}$ and families of smoothing operators along the fibers of $\pi$ is $a^{*}$-algebra isomorphism.

From now on we will think of the symbol of an operator in $\mathscr{R}$ as such a family of smoothing operators. Other symbol calculus rules will be quoted as needed. Note that the action of $\mathbb{R}^{+}$on $\Sigma$ induces an action of $\mathbb{R}^{+}$on $W$ and the natural projection $\Sigma \rightarrow W$ is equivariant. Thus one can speak in an obvious way of symbols homogeneous of a given degree. We denote by $\mathscr{R}^{m}$ the subspace of $\mathscr{R}$ consisting of operators which are homogeneous of degree $m$.

With this symbol calculus at hand, we proceed to construct $\Pi . \forall w \in W$ its symbol will be the rank-one projector onto an element $\mu^{w} \in C^{\infty}\left(\left|T F^{w}\right|^{1 / 2} \otimes \mathscr{L}^{w}\right)$. The definition of $\mu$ is as follows. Trivialize $\left|T F^{w}\right|^{1 / 2}$ by the half-density dual to the Hamilton vector field of $q$. Using the action of $S_{T}^{1}$ on $F^{w}$, one gets an identification

$$
C^{\infty}\left(\left|T F^{w}\right|^{1 / 2} \otimes \mathscr{L}^{w}\right) \cong \mathscr{F}^{w}=\left\{u \in C^{\infty}(\mathbb{R}) ; \forall k \in \mathbb{Z} u(\theta+k T)=e^{i k \pi \sigma / 2} u(\theta)\right\},
$$

with the pre-Hilbert space structure $\langle u, v\rangle=\int_{0}^{T} u \bar{v} d \theta$. Define $\mu^{w}$ as that section corresponding to the function $\theta \mapsto T^{-1 / 2} e^{i \pi \sigma \theta / 2 T}$.

Proposition 4.4 [8, Proposition 4.1]. There exists a self-adjoint projection, $\Pi$, which commutes with $Q$, is in the ring $\mathscr{R}$, and has symbol the family of rank-one projectors $\mu \otimes \mu$. Moreover, in the circular symmetry setting, $\Pi$ can be chosen as to commute with $A$.

Proof. In Proposition 4.1 of [8] it is shown that there exists an orthogonal projection with this symbol. The construction there will produce $\Pi$ commuting 
with $Q$ and $A$ if we can find a self-adjoint operator in $\mathscr{R}$ with symbol $\mu \otimes \mu$ and commuting with $Q$ (respectively $A$ ). We now look for such an operator of the form $\varphi(Q)$.

Claim. $\forall \hat{\varphi} \in C_{0}^{\infty}(\mathbb{R}), \varphi(Q) \in \mathscr{R}^{0}$. Moreover, with the identification (52), its symbol along the fiber $\mathscr{F}^{w}$ is the operator

$$
S(u)(\theta)=(2 \pi)^{-1 / 2} e^{i \sigma \pi \theta / 2 T}\left(u_{0} * \tilde{\varphi}\right)
$$

where $u_{0}(\theta)=u(\theta) \exp (-i \sigma \pi \theta / 2 T)$ is $T \mathbb{Z}$ periodic, * is convolution in $S_{T}^{1}$ and

$$
\tilde{\varphi}(x)=\sum_{k \in \mathbb{Z}} \hat{\varphi}(-x+k T) .
$$

Proof of the Claim. For the first part, see [20]. The Fourier integral operator $\exp (i t Q)$ acts on the right and on the left on the ring $\mathscr{R}$. At the symbolic level, the action is by translation, $u(\theta) \mapsto u(\theta+t)$. The symbol of $\varphi(Q)$ maps $u \in \mathscr{F}^{w}$ to

$$
\theta \mapsto(2 \pi)^{-1 / 2} \int_{\mathbb{R}} \hat{\varphi}(t) u(\theta+t) d t,
$$

as is seen from the equation $\varphi(Q)=(2 \pi)^{-1 / 2} \int e^{i t Q} \hat{\varphi}(t) d t$. The right-hand side of (55) is easily seen to equal (53).

Thus $\varphi(Q)$ will have for symbol $\mu \otimes \mu$ if $\forall u \in \mathscr{F}^{w}$

$$
u_{0} * \tilde{\varphi}=\frac{(2 \pi)^{1 / 2}}{T} \int_{0}^{T} u_{0}(\theta) d \theta
$$

By the Poisson summation formula, it is enough that

$$
\forall k \in \mathbb{Z}-\{0\} \varphi\left(\frac{4 k \pi}{2 T}\right)=0 \quad \text { and } \quad \varphi(0)=2 \pi T,
$$

and one can take for example a function of the form

$$
\varphi(x)=f(x) \frac{\sin (T x)}{x}, \quad f(0)=2 \pi,
$$

where $f(x)$ has a smooth and compactly supported Fourier transform. The Fourier transform of such a $\varphi$ is the convolution of the characteristic function of an interval of length $T$ and a compactly supported smooth function. Hence the resulting operator is indeed a smoothed-out version of (49).

Next we show that modulo smoothing operators $\Pi$ is the projection into the kernel of a perturbation of $Q$. Let $Q$ and $\Pi \in \mathscr{R}$ be as above (in particular: $\Pi Q=Q \Pi$, the symbol of $\Pi$ is $\mu \otimes \mu$, and $q=q_{\text {sub }}=0$ on $\Sigma$ ).

Proposition 4.5. There is a self-adjoint, pseudodifferential operator of order $(-1), R$, such that

$$
\left(Q-\frac{\sigma \pi}{2 T} I-R\right) \circ \Pi=0 .
$$

In the circular symmetry setting, $R$ can be chosen to commute with $A$.

Proof. The second part follows by averaging with respect to $\exp (i \theta A)$. We sketch the proof of the first statement, which is a slight strengthening of $[8$, Theorem 5.6]. The symbol of $Q \Pi$ is the symbol of $\Pi$ followed by the operator $-i \Xi_{q}$, by $[8$, 
Proposition 3.2]); hence it is the operator $u \mapsto \frac{\sigma \pi}{2 T}\langle u, \mu\rangle \mu$. Thus $Q_{1} \Pi \in \mathscr{R}^{-1}$, where $Q_{1}=Q-\frac{\sigma \pi}{2 T} I$. Since $\left(Q_{1} \Pi\right) \Pi=Q_{1} \Pi$, the symbol of $Q_{1} \Pi$ is of the form $\mu_{1} \otimes \mu$, where $\mu_{1}$ is a section of $|T F|^{1 / 2}$ homogeneous of degree -1 . The symbol calculus implies that, since $\Pi$ commutes with $Q_{1}, \mu_{1}$ is of the form $\mu_{1}=\varrho \mu$, where $\varrho$ is a function on $\Sigma$ homogeneous of order -1 . Let $R_{1}$ be a pseudodifferential operator of order -1 whose symbol agrees with $\varrho$ on $\Sigma$. The symbol calculus shows that $\left(Q_{1}-R_{1}\right) \Pi$ is of order lower than $Q_{1} \Pi$. Now continue exactly as in the proof of Theorem 5.6 [8].

Let us define $\mathscr{Q}=Q-\frac{\sigma \pi}{2 T} I-R$.

Theorem 4.6 $[10,7]$. There is a bounded operator, $L$, such that

$$
L \circ \mathscr{Q}=I-\Pi+S \text {, }
$$

where $S$ is a smoothing operator.

Proof. See [10, Theorem 7.2] and the discussion that follows it. The operator $L$ is constructed symbolically using the calculus of Fourier integral operators associated to two intersecting lagrangians, as developed in [10]. Thus its Schwartz kernel is in a space $I^{p, l}(M \times M ; \Delta, \mathscr{C})$ of Guillemin and Uhlmann, where $\Delta$ is the diagonal, $\Delta \subset X \times X\left(X=T^{*} M-\{0\}\right)$, and $\mathscr{C}$ is the bicharacteristic relation. Elements of this space are distributions that, microlocally near $\Delta-(\Delta \cap \mathscr{C})$ are in the Hörmander space $I^{p+l}(M \times M, \Delta)$, while near $\mathscr{C}-(\Delta \cap \mathscr{C})$ are in $I^{p}(M \times M, \mathscr{C})$. Matching orders in (60) near these two manifolds, we get $p=-1 / 2=l$ [the Fourier integral operator $\Pi$ has kernel in $I^{-1 / 2}(M \times M ; \mathscr{C})$; this can be seen from the equation $\Pi \circ \Pi=\Pi$ and the fact that the composition of $\Pi$ with itself has excess equal to one]. By Theorem 3.3 of [7] and a microlocal partition of unity, since

$$
\max (p+1 / 2, p+l)=0,
$$

the operator $L$ is bounded in $L^{2}(M)$.

One can easily show that the image of $\Pi$ has finite codimension in the kernel of 2; cf. [8], remark after Proposition 6.7.

Corollary 4.7. Zero is an isolated point in the spectrum of 2 .

Proof. Let $\mu_{j}$ be a sequence of non-zero eigenvalues of 2 . By (59), we can write

$$
\mathscr{2}\left(u_{j}\right)=\mu_{j} u_{j},
$$

with the $u_{j}$ of norm one and in $\operatorname{Im}(\Pi)^{\perp}$. Applying $L$ to $(61)$, taking the inner product with $u_{j}$ and using (60), get

$$
C\left|\mu_{j}\right| \geqq\left|1+\left\langle S\left(u_{j}\right), u_{j}\right\rangle\right|,
$$

$\forall j$, with $C$ the norm of $L$. Since the operator $S$ is compact, zero is not an accumulation point of the $\mu_{j}$.

$B$. Back in the circular symmetry setting, we now consider the case when the trajectory through each point on $B_{E}$ is periodic. More precisely, assume: (i) $E$ is a regular value of $H$, (ii) $B_{E}$ is connected, and (iii) each point in $B_{E}$ is periodic with minimal period $T$, with $T>0$ fixed. For each $b \in B_{E}$, let $\gamma_{b}$ denote the trajectory 
$\phi_{t}(b), t \in[0, T]$. If $\exp \left(i \int_{\gamma} \alpha\right)$ denotes the holonomy of a closed curve $\gamma$ with respect to the natural connection on the circle bundle $\{a=1\} \rightarrow B$, the mapping $B_{E} \rightarrow S^{1}$ defined by

$$
b \mapsto \exp \left(i \int_{\gamma_{b}} \alpha\right)
$$

is locally constant [11, Lemma 4.5]. Since we are assuming that $B_{E}$ is connected, this map is constant. We will now assume that the trajectories $\gamma_{b}$ satisfy the BohrSommerfeld quantization condition:

$$
\exp \left(i \int_{\gamma_{b}} \alpha\right)=1
$$

By the constancy of (63), if this happens for one $b$ it happens for all $b$, and implies that the only singularity of $Y$ is at $z=1$. This assumption, or at least the assumption that the left-hand side of (64) be a root of unity, can be seen to be essential for what follows below.

Theorem 4.8. Under the previous assumptions, including (64), there are constants $C_{1}, C_{2}>0$ and an integer $\sigma$ such that, if $m$ is sufficiently large,

$$
\left\{j ;\left|\lambda_{j}(m)-m E-\pi \sigma / 2 T\right| \leqq C_{1}\right\} \subset\left\{j ;\left|\lambda_{j}(m)-m E-\pi \sigma / 2 T\right| \leqq C_{2} m^{-1}\right\} .
$$

The integer $\sigma$ is the Maslov index of the null bicharacteristics of the real-principal type operator $Q=P-E A$, which are all closed with minimal period T. Furthermore, the cardinality of the set on the right-hand side of $(65)$ is, for large $m$, a polynomial function of $m$ of degree $n-1$ and leading coefficient the Liouville measure of $B_{E}$ divided by $(2 \pi)^{n}$.

We will refer to the set on the right-hand side of (65) as the $m^{\text {th }}$ cluster in the spectrum. The polynomial giving the number of eigenvalues in the $m^{\text {th }}$ cluster is very closely related to the Riemann-Roch polynomial $p(x)$ (as defined in [1]) of the symplectic manifold

$$
Y=B_{E} /\left(S_{T}^{\mathbf{1}}\right)
$$

where we let $S_{T}^{1}$ act on $B_{E}$ via the flow $\phi_{t}$. $B_{E}$ is a smooth closed manifold of dimension $2(n-1)$, which inherits a natural symplectic structure induced from the restriction to $B_{E}$ of the symplectic form on $B$. The Riemann-Roch polynomial $\mathfrak{p}(x)$ associated to $Y$ has degree $n-1$ and leading coefficient the Liouville measure of $Y$.

Theorem 4.9. If (64) holds, there is an integer $m_{0}$ such that if $m$ is large enough, the cardinality of the right-hand side of (65) is equal to $\mathrm{p}\left(m+m_{0}\right)$.

Proof of Theorem 4.8. We first notice that the operator $Q=P-E A$ is of real principal type, and all its null bicharacteristics are periodic of minimal period $T$. (Proof: Zero is a regular value of $q=p-E a$ because $E$ is a regular value of $H$. By the periodicity assumption, for any $x \in \Sigma \cap\{a=1\}$ there is a $\theta \in \mathbb{R}$ such that one has

$$
\exp \left(\theta \Xi_{a}\right) \circ \exp \left(T \Xi_{p}\right)(x)=x,
$$

where $\Xi_{a}$, respectively $\Xi_{p}$, is the Hamilton vector field of $a$, respectively $p$. By Theorem 4.3 in [11], and (64), $E T+\theta \in 2 \pi \mathbb{Z}$. Since (67) continues to hold if we add to $\theta$ a multiple of $2 \pi$, it is clear that we can take $\theta=-E T$. This means by (67) that

$$
\exp \left(T \Xi_{p-E a}\right)(x)=x \text {. }
$$


This proves the periodicity of the null bicharacteristics on $\{a=1\}$; the periodicity of the other ones follows by homogeneity.)

Write

$$
P-E A-\frac{\pi \sigma}{2 T} I=\mathscr{Q}+R
$$

as in Theorem (4.1). Let $\mathscr{H}=\bigoplus_{m} \mathscr{H}_{m}$ be the eigenspace decomposition of $A$. Since $R$ is of order -1 , the composition $A R$ is bounded in $L^{2}(M)$. Let $C_{2}$ be its norm. It is clear that the norm of the restriction of $R$ to $\mathscr{H}_{m}$ is bounded by $C_{2} m^{-1}$. By standard perturbation theory, (e.g. [17, Theorem 4.10]), one has

$$
\operatorname{dist}\left(\operatorname{Spec}\left[Q_{m}-(\pi \sigma / 2 T) I\right], \operatorname{Spec}\left(\mathscr{Q}_{m}\right)\right) \leqq C_{2} m^{-1},
$$

where Spec stands for "spectrum," and $Q_{m}$, respectively $\mathscr{Q}_{m}$, denote the restriction of $Q=P-E A$, respectively 2 , to $\mathscr{H}_{m}$. By Corollary 4.7 , there is a constant $C>0$ such that there are no non-zero eigenvalues of 2 in $[-C, C]$. Since the spectrum of $Q_{m}$ is the set of eigenvalues $\left\{\lambda_{j}(m)-m E ; j \geqq 1\right\}$, the first part of Theorem 4.8 follows, with $C_{1}$ a constant slightly smaller than $C$.

One can push the analysis further, along the lines of the theory of "band asymptotics", $[4,22]$. Let $\varrho$ be the principal symbol of $R$. Since it Poisson commutes with $A$, there is a function on $B_{E}$ corresponding to it; we will also denote this function by $\varrho$. Let $\mu$ denote Liouville measure on $B_{E}$, and $J(m)$ the set of indices of eigenvalues in the $m^{\text {th }}$ cluster. The eigenvalues $\lambda_{j}(m)$ in the $m^{\text {th }}$ cluster are those that split off from $\operatorname{Ker} \mathscr{2}_{m}$, and so for large $m, \# J(m)=\operatorname{dim}\left(\operatorname{Ker} \mathscr{2}_{m}\right)$.

Proposition 4.10. For every $f \in C^{\infty}(\mathbb{R})$, there is an asymptotic expansion

$$
\sum_{j \in J(m)} f\left(m\left(\lambda_{j}(m)-m E-\pi \sigma / 2 T\right)\right) \sim \sum_{j \geqq 0} m^{n-1-j} \beta_{j}(f),
$$

where

$$
\beta_{0}(f)=\int_{\boldsymbol{B}_{\boldsymbol{E}}} f \circ \varrho d \mu .
$$

Proof. Very succinctly, one forms the following trace:

$$
\operatorname{Tr}\left(f(A R) e^{i \theta A} \Pi\right) \text {. }
$$

The operator $f(A R)$ (defined by the spectral theorem) is a classical pseudodifferential operator of order zero, so the construction of (73) as a distributional function of $\theta$ can be carried out within the theory of Fourier integral operators. On the other hand, (69) and the fact that $\operatorname{Im} \Pi$ has finite codimension in Ker 2 imply that modulo a $C^{\infty}$ function the distribution (73) is equal to

$$
\sum_{m \in \mathbb{Z}} e^{i m \theta} \sum_{j \in J(m)} f\left(m\left(\lambda_{j}(m)-m E-\pi \sigma / 2 T\right)\right)
$$

The proposition follows from these facts and a calculation of the symbol of (73).

We do not have an explicit description of $\varrho$. However, this proposition implies that the multiplicity of the $m^{\text {th }}$ cluster is a polynomial function of $m$, with the properties stated at the end of Theorem 4.8. Indeed, take $f$ in (71) to be identically equal to one. The proposition implies that the function $d(m)=\# J(m)$ admits an asymptotic expansion in decreasing powers of $m$, with the correct leading order 
term. Since for every integer, $m, d(m)$ is an integer, this implies that $d(m)$ is a polynomial in $m$ [16, Lemma 29.2.3].

Theorem 4.9, now follows directly from results of Boutet de Monvel and Guillemin in Sect. 14 of [1], particularly Theorem 14.10, applied to the symplectic manifold (66).

Let us now return to the semi-classical problem and consider $-\hbar^{2} A+V$ on a compact Riemannian manifold. Interpreting $\hbar^{-1}$ as an eigenvalue of $-i \partial_{\theta}$ and applying Theorems 4.8 and 4.9 leads to Theorem 2.3 on a compact manifold. To prove Corollary 2.5, again on a compact manifold, consider $\hbar^{-1}$ as eigenvalue of $A=-i R \partial_{\theta}$ on $S^{1}$ and form again $Q=P-E A$, with $P^{2}=-\Delta+A^{2} V$. One easily checks that the null bicharacteristics of $Q$ are periodic iff $R \alpha \in 2 \pi \mathbb{Z}$. Now choose $R=2 \pi / \alpha$. Then Theorem 4.8 holds with $m$ replaced by $2 \pi m / \alpha$.

\section{The Trace Formula for Schrödinger Operators on $\mathbb{R}^{n}$}

We will first compare (5) with the analogous expression where the $\lambda_{j}(h)$ are replaced by the Dirichlet eigenvalues of $S(\hbar)$ on a bounded open neighborhood of the set $\{x: V(x) \leqq E\}$. We are able to do this using the following result of Helffer and Sjöstrand [13].

Let $M$ be either $\mathbb{R}^{n}$ or a compact Riemannian manifold. Let $\Delta$ denote the Laplace-Beltrami operator on $M$ and let $V \in C^{\infty}(M), V>0$. Let $E \in \mathbf{R}$, $E<\liminf _{|x| \rightarrow \infty} V(x)$ if $M$ is non-compact. Write

$$
V^{-1}(-\infty, E]=W_{1} \cup W_{2} \cup \ldots \cup W_{N},
$$

where the "wells" $W_{j}$ are connected, compact and pairwise disjoint. Let $\Omega_{k} \supset W_{k}$ be bounded connected open subsets of $M$ with $C^{\infty}$ boundary, such that $\Omega_{k}$ is disjoint with an open neighborhood of $W_{j}$ for all $j \neq k$. Let $S(\hbar)_{\Omega_{k}}$ denote the Dirichlet realization of $S(\hbar)$ on $\Omega_{k}$ [so that eigenfunctions of $S(\hbar)_{\Omega_{k}}$ are eigenfunctions of $S(\hbar)$ which satisfy the Dirichlet boundary condition on $\left.\partial \Omega_{k}\right]$.

Let $E<\underline{V}_{\infty}$ and let $I(\hbar)$ denote an interval $I(\hbar)=[E-\alpha(\hbar), E+\beta(\hbar)]$ with $\alpha(\hbar), \beta(\hbar) \rightarrow 0$ as $\hbar \rightarrow 0$. Let the function $e(\hbar)>0$ satisfy $\log e(\hbar)=o\left(\hbar^{-1}\right), \hbar \rightarrow 0$.

Theorem 5.1 (Helffer and Sjöstrand [13], cf. also Helffer [12]). Suppose that $S(\hbar)_{\Omega_{k}}$ has no spectrum in the intervals $[E-\alpha(\hbar)-e(\hbar), E-\alpha(\hbar)]$ and $[E+\beta(\hbar), E+\beta(\hbar)+e(\hbar)]$. Then for sufficiently small $\hbar$, there exists a bijection

$$
b=b_{\hbar}: \operatorname{Spec} S(\hbar) \cap I(\hbar) \rightarrow \bigcup_{k=1}^{N} \operatorname{Spec} S(\hbar)_{\Omega_{k}} \cap I(\hbar)
$$

(disjoint union on the right) such that for some $\sigma>0$,

$$
b_{\hbar}(\lambda)-\lambda=O\left(e^{-\sigma / \hbar}\right)
$$

( $\sigma$ can be taken arbitrarily close to the smallest Agmon distance between the wells, but that will not be needed here).

We will need the following technical lemma.

Lemma 5.2. Let $S(\hbar)=-\hbar^{2} \Delta+V$ be a Schrödinger operator on $M$ and let $\varphi \in \mathscr{S}(\mathbb{R})$. We let the variable $\mu$ range over $\operatorname{Spec} S(\hbar)$. Let $\varepsilon>0$. 
(i) If $M$ is compact and $V(x)>0$ on $M$, then

$$
\left|\frac{\sqrt{\mu}-E}{\hbar}\right|_{\geqq \hbar-\varepsilon} \cdot\left|\varphi\left(\frac{\sqrt{\mu}-E}{\hbar}\right)\right|=O\left(\hbar^{\infty}\right) \text { as } \hbar \rightarrow 0 .
$$

(ii) In case $M$ is $\mathbb{R}^{n}, E^{2}<\liminf _{|x| \rightarrow \infty} V(x)$ and $\varepsilon<1$, we have

$$
\sum_{\hbar^{-\varepsilon} \leqq}\left|\frac{\sqrt{\mu-E}}{\hbar}\right|_{\leqq 2 \hbar^{-\varepsilon}}\left|\varphi\left(\frac{\sqrt{\mu}-E}{\hbar}\right)\right|=O\left(\hbar^{\infty}\right), \quad \hbar \rightarrow 0 .
$$

In the proof of this lemma we will use the following two rough estimates:

(a) In the situation of Lemma 5.2(i) there exists an $N_{0} \in \mathbf{N}$ and a constant $C$ such that

$$
\#\left\{\mu \in \operatorname{Spec} S(\hbar): \frac{\sqrt{\mu}-E}{\hbar} \leqq K\right\} \leqq C \cdot\left(\frac{K}{\hbar}\right)^{N_{0}} .
$$

(b) In case $M$ is $\mathbb{R}^{n}$ and $K<\underline{V}_{\infty}$, there exist $N_{0}$ and $C$ (which may depend on $K$ here) such that

$$
\#\{\lambda \in \operatorname{Spec} S(\hbar): \lambda \leqq K\} \leqq C \cdot \hbar^{-N_{0}}
$$

In fact, one can take $N_{0}=n$ in both these estimates. Estimate (78) follows for example by comparing $S(h)$ with $-\Delta$ and using the classical Weyl estimate; $(79)$ can be proved using min-max and Dirichlet-von Neumann bracketing.

Proof of Lemma 5.2. (i) Since $\varphi \in \mathscr{P}(\mathbb{R}),|\varphi(t)| \leqq C_{N}|t|^{-N}$ for $|t| \geqq 1, N=1,2, \ldots$. Hence the sum in (76) is bounded by

$$
\begin{aligned}
& \sum_{k=\left[\hbar^{-\varepsilon}\right]}^{\infty} \sum_{k \leqq\left|\frac{\sqrt{\mu}-E}{\hbar}\right| \leqq k+1}\left|\varphi\left(\frac{\sqrt{\mu}-E}{\hbar}\right)\right| \\
& \leqq C_{N} \cdot \sum_{k=\left[\hbar^{-\varepsilon}\right]}^{\infty} k^{-N} \cdot \#\left\{\mu \in \operatorname{Spec} S(\hbar):\left|\frac{\sqrt{\mu}-E}{\hbar}\right| \leqq k+1\right\} \\
& \leqq C_{N} \sum_{k=\left[\hbar^{-\varepsilon}\right]}^{\infty} k^{-N} \cdot k^{N_{0}} \cdot \hbar^{-N_{0}}
\end{aligned}
$$

[where we used (78)]

$$
\leqq C_{N} \cdot \hbar^{-N_{0}} \cdot\left(\hbar^{-\varepsilon}\right)^{N_{0}-N+1}
$$

for sufficiently large $N \in \mathbb{N}$.

(ii) Proceed as above, but now note that since the sum over $k$ only ranges from $\left[\hbar^{-\varepsilon}\right]$ till $\left[2 \hbar^{-\varepsilon}\right]$ and $\varepsilon<1$,

$$
\#\left\{\mu \in \operatorname{Spec} S(\hbar):\left|\frac{\sqrt{\mu}-E}{\hbar}\right| \leqq k+1\right\} \leqq \#\{\mu: \sqrt{\mu} \leqq E+\eta\}
$$

for sufficiently small $\hbar$, where $\eta>0$ is some number such that $(E+\eta)^{2}<V_{\infty}$. By (79) above, this is $O\left(\hbar^{-N_{0}}\right)$.

Proof of Theorem 2.1. First, we reduce the problem to a disjoint sum of Dirichlet eigenvalue problems by applying Theorem 5.1 with suitable $\alpha(\hbar)$ and $\beta(\hbar)$. 
Let $\eta$ be some number such that $(E+\eta)^{2}<\underline{V}_{\infty}$. Recall that the number of eigenvalues of $S(\hbar)_{\Omega_{k}}$ smaller than $(E+\eta)^{2}$ is $O\left(\hbar^{-N_{0}}\right)$. It follows that for any $\delta>0$ there exist $\alpha(\hbar), \beta(\hbar)$ such that $2 E \hbar^{\delta}<\alpha(\hbar), \beta(\hbar)<4 E \hbar^{\delta}$ and such that $S(\hbar)_{\Omega_{\gamma}}$ has no

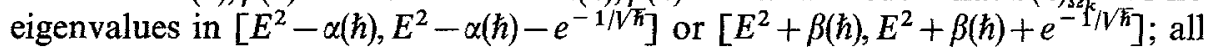
this for $\hbar$ sufficiently small. We will take $\delta=1-\varepsilon, \varepsilon$ as in (4).

Now in (5) we sum over those eigenvalues $\lambda$ of $S(\hbar)$ such that $E^{2}-2 E \hbar^{\delta}+\hbar^{2 \delta} \leqq \lambda$ $\leqq E^{2}+2 E \hbar^{\delta}+\hbar^{2 \delta}$. We may replace this by a summation over $\lambda$ 's in $I(\hbar):=\left[E^{2}-\alpha(\hbar), E^{2}+\beta(\hbar)\right]$, since by Lemma $5.2($ ii) the contribution of the $\lambda$ 's in the difference of these two sets is $O\left(\hbar^{\infty}\right)$. By Theorem 5.1,

$$
\begin{aligned}
& \left|\sum_{\lambda \in \operatorname{Spec} S(\hbar) \cap I(\hbar)} \varphi\left(\frac{\sqrt{\lambda}-E}{\hbar}\right)-\sum_{k=1}^{N} \sum_{\mu \in \operatorname{Spec} S(\hbar)_{\Omega_{k}} \cap I(h)} \varphi\left(\frac{\sqrt{\mu}-E}{\hbar}\right)\right| \\
& \leqq \max \left|\varphi^{\prime}\right| \cdot \sum_{\lambda \in \operatorname{Spec} S(\hbar) \cap I(\hbar)}\left|\frac{\sqrt{\lambda}-\sqrt{b_{h}(\lambda)}}{\hbar}\right| \\
& \leqq \max \left|\varphi^{\prime}\right| \cdot \frac{e^{-\sigma / 2 \hbar}}{\hbar} \cdot \# \operatorname{Spec} S(\hbar) \cap\left(-\infty,(E+\eta)^{2}\right]
\end{aligned}
$$

for $\hbar$ sufficiently small; here we used that $E>0$ so that 0 is not in $I(\hbar)$ for $\hbar$ sufficiently small. By (79) this last term is $O\left(h^{-N_{0}} e^{-\sigma / 2 \hbar}\right)=O\left(\hbar^{\infty}\right)$.

Now we will use the same argument in the other direction (from Dirichlet to global eigenvalue problem) but this time on a compact manifold. One easily constructs a Riemannian manifold $M$ and a $\widetilde{V} \in C^{\infty}(M)$ such that

(i) $\Omega_{k} \subset M$ and $\{x \in M: \tilde{V}(x) \leqq E\}=\{V \leqq E\} \subset \bigcup_{k} \Omega_{k}$. (ii) The Riemannian metric of $M$ restricted to a neighborhood of $\bigcup_{k} \Omega_{k}$ is
Euclidean.

(For example, one can take $M$ equal to the double of a connected, bounded, smoothly bounded neighborhood of $\bigcup_{k} \Omega_{k}$ and construct a suitable metric using a partition of unity.)

Let $\tilde{S}(\hbar)=-\hbar^{2} \Delta_{M}+\tilde{V}, \Delta_{M}$ being the Laplace-Beltrami operator on $M$. Repeating the arguments above, we see that

$$
\sum_{\substack{\mu \in \operatorname{Spec} \tilde{S}(h) \\|\sqrt{\mu}-E| \leqq \hbar^{1-\varepsilon}}} \varphi\left(\frac{\sqrt{\mu}-E}{\hbar}\right)=\sum_{k} \sum_{\mu \in \operatorname{Spec} S(\hbar)_{\Omega_{k}} \cap I(\hbar)} \varphi\left(\frac{\sqrt{\mu}-E}{\hbar}\right)+O\left(\hbar^{\infty}\right) .
$$

By Lemma 5.2(i), (80) differs only $O\left(\hbar^{\infty}\right)$ from

$$
\sum_{\mu \in \operatorname{Spec} \tilde{\mathcal{S}}(\hbar)} \varphi\left(\frac{\sqrt{\mu}-E}{\hbar}\right) \text {. }
$$

This completes the proof, since the asymptotic expansion of (81) follows from Theorem 3.1.

Proof of Theorem 2.2. Let $E^{2}<\underline{\infty}_{\infty}$. For sufficiently small $\hbar$,

$$
N_{E, c}(h)=\#\left\{j \in J_{E, \varepsilon}(h):\left|\sqrt{\lambda_{j}(h)}-E\right| \leqq c h\right\} .
$$

Now repeat the proof of Theorem 3.2, starting from the trace formula for $\mathbb{R}^{n}$ (and replacing $m$ by $\hbar^{-1}$ everywhere).

Proof of Theorem 2.3 and Corollary 2.4. Use Theorem 5.1 to reduce to the compact case, as in the proof of the trace formula (details left to the reader). 
Note added in proof. In joint work with Thierry Paul we have recently realized that one can remove from the trace formula the square roots of the quantum and classical Hamiltonians. Details will appear elsewhere.

Acknowledgements. We wish to thank Thierry Paul, Jeffrey Rauch, Heinz Siedentop, Gunther Uhlmann, and André Voros for helpful discussions. Most of this research was carried out in the spring of 1989, when both authors were at Princeton University. One of us (R.B.) gratefully acknowledges the support of the Netherlands Organization for Scientific Research, through a NATO fellowship.

\section{References}

1. Boutet de Monvel, L., Guillemin, V.. The spectral theory of Toeplitz operators. Ann. Math. Stud. vol. 99. Princeton, NJ: Princeton University Press 1981

2. Chazarain, J.: Spectre d'un hamiltonien quantique et mécanique classique. Comm. PDE 6, 595-644 (1980)

3. Colin de Verdière, I.: Spectre conjoint d'opérateurs pseudodifférentiels qui commutent. Duke Math. J. 46, 169-182 (1979)

4. Colin de Verdière, I.: Sur le spectre des opérateurs elliptiques à bicharacteristiques toutes périodiques. Comment. Math. Helv. 54, 508-522 (1979)

5. Duistermaat, J., Guillemin, V.: The spectrum of positive elliptic operators and periodic bicharacteristics. Invent. Math. 29, 39-79 (1975)

6. Gutzwiller, M.: Periodic orbits and classical quantization conditions. J. Math. Phys. 12, 343-358 (1971)

7. Greenleaf, A., Uhlmann, G.: Estimates for singular Radon transforms and pseudodifferential operators with singular symbols. Preprint

8. Guillemin, V., Sternberg, S.: Some problems in integral geometry and some related problems in micro-local analysis. Am. J. Math. 101 (4), 915 955 (1979)

9. Guillemin, V., Sternberg, S.: Homogeneous quantization and multiplicities of group representations. J. Funct. Anal. 47 (3), 344-380 (1982)

10. Guillemin, V., Uhlmann, G.: Oscillatory integrals with singular symbols. Duke Math. J. 48, 251-267 (1981)

11. Guillemin, V., Uribe, A.: Circular symmetry and the trace formula. Invent. Math. 96, 385423 (1989)

12. Helffer, B.: Semi-classical analysis for the Schrödinger operator and applications. Springer Lecture Notes vol. 1336. Berlin, Heidelberg, New York: Springer 1988

13. Helffer, B., Sjöstrand, J.: Multiple wells in the semi-classical limit I. Comm. PDE 9(4), 337-408 (1984)

14. Helffer, B., Robert, D.: Comportement semi-classique du spectre des hamiltoniens quantiques elliptiques. Ann. Inst. Fourier tome XXXI, Fax 3, 169-223 (1981)

15. Helffer, B., Robert, D.: Comportement semi-classique du spectre des hamiltoniens quantiques hypoelliptiques. Ann. Ecole Normale Superiore di Pisa 9, 405-431 (1982)

16. Hörmander, L.: The analysis of linear partial differential operators IV. Berlin, Heidelberg, New York: Springer 1985

17. Kato, T.: Perturbation theory for linear operators. 2nd edition. Berlin, Heidelberg, New York: Springer 1980

18. Petkov, V., Robert, D.: Asymptotique semi-classique d'Hamiltoniens quantiques et trajectoires classiques périodiques. Comm. PDE 10 (4) (1985)

19. Robert, D.: Autour de l'approximation semi-classique. Progress in Mathematics vol. 68. Boston: Birkhäuser 1987

20. Taylor, M., Uribe, A.: Semi-classical spectra of gauge fields, in preparation

21. Voros, A.: Schrödinger equation from $O(\hbar)$ to $o\left(\hbar^{\infty}\right)$. In: Path integrals from meV to Mev, proceedings of the VII Bielefeld encounters in Physics and Mathematics. Gutzwiller, M.C., Inomata, A., Klauder, J.R., Streit, L. (eds.). Singapore: World Scientific 1986

22. Weinstein, A.: Asymptotics of eigenvalue clusters for the Laplacian plus a potential. Duke Math. J. 44, 883-892 (1977) 